\title{
Homotopy composition of cospans
}

\author{
Joachim Kock and David I. Spivak
}

\begin{abstract}
It is well known that the category of finite sets and cospans, composed by pushout, contains the universal special commutative Frobenius algebra. In this note we observe that the same construction yields also general commutative Frobenius algebras, if just the pushouts are changed to homotopy pushouts.
\end{abstract}

\section{Introduction}

A Frobenius algebra, or more generally, a Frobenius object in a monoidal category [6], is special when the comultiplication followed by the multiplication is the identity map. A symmetric monoidal category containing the universal special commutative Frobenius algebra was exhibited by Lack [7], and independently by Rosebrugh, Sabadini and Walters 8 ] in their study of general processes: it is the category whose objects are finite sets and whose morphisms are cospans; the composition of cospans is given by pushout. Special commutative Frobenius objects and cospan categories have also been studied recently by Baez and Fong [2] in the context of electrical network theory, and Coya and Fong [3] have shown that the category of jointly surjective cospans contains the universal extraspecial commutative Frobenius algebra, meaning that also unit-followed-by-counit is required to be the identity 1

In this note we observe that "specialness" is a result of the simple-minded nature of pushouts in the category of sets: if one replaces pushouts by homotopy pushouts, the resulting cospan category contains the universal commutative Frobenius object, rather than the special one. To make sense of the homotopy pushout, a homotopical setting is required. The minimal setting in which the construction works is that of finite 1-dimensional CW complexes up to homotopy, i.e. topological graphs, the endpoints of the cospans being still just finite sets. Graph cospans like this have a long history in the theory of graph rewriting (an early explicit reference being [5]), but hitherto, as far as we know, only set-theoretical pushouts have been considered.

In light of the growing importance of homotopy viewpoints in modern mathematical sciences, we wonder whether the finer composition of cospans, provided by the homotopy pushout, may be of relevance in the various application areas of the cospan model. We leave the investigation of these issues to the experts in the respective fields.

\section{Cobordisms versus cospans}

To put the cospan construction in perspective, and to explain its homotopy version, we exploit the standard result that the universal commutative Frobenius algebra lives in the symmetric monoidal category $\mathbf{2 C o b}$ of 2-dimensional cobordisms [6. Theorem 3.6.19]. (A corollary is the notorious equivalence between $2 \mathrm{~d}$ topological quantum field theories and commutative Frobenius algebras.)

The result essentially amounts to writing the axioms for commutative Frobenius algebras in graphical language, and observing that they precisely express evident topological properties of cobordisms. We shall not repeat the arguments (nor even the axioms) here, but content ourselves to list the graphical building blocks corresponding to multiplication and unit, comultiplication and counit, and the corre-

\footnotetext{
${ }^{1}$ In the context of conformal field theory (see for example [4), "extraspecial" is called just "special".
} 
sponding cospans:
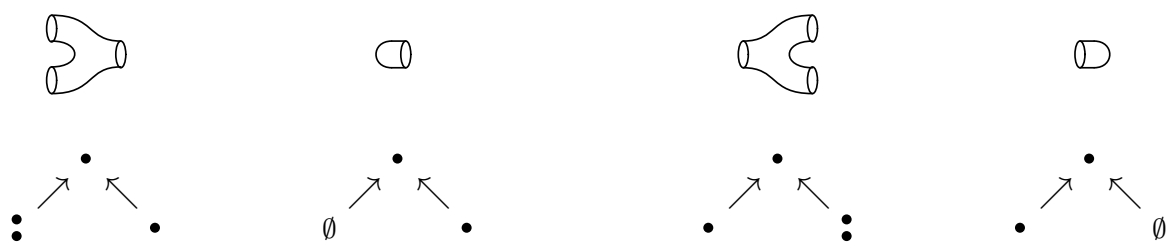

The analogy shown in (11) between $2 \mathrm{~d}$ cobordisms and cospans $A_{0} \rightarrow X \leftarrow A_{1}$ of finite sets is clear: $A_{0}$ corresponds to the set of in-boundaries, $A_{1}$ corresponds to the set of out-boundaries, and $X$ corresponds to the set of connected components of a cobordism. Composition of cobordisms is given by gluing at the boundaries (in fact a pushout operation); cospans compose by pushout.

However, the analogy breaks down quickly in more complicated situations, because the set $X$ does not contain information about the genus of each component of a cobordism. The crucial difference can be pinpointed to the following basic composition of cospans of sets, expressing precisely the axiom characterizing special Frobenius algebras among all Frobenius algebras:

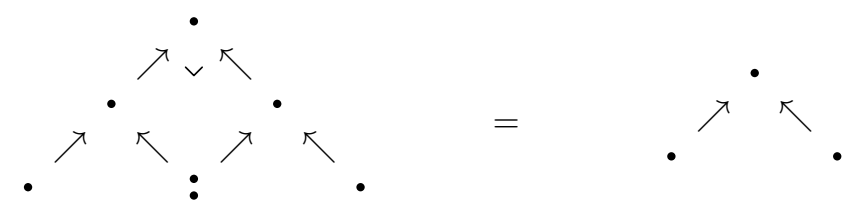

We see that cospans (in the category of sets) cannot render the idea of a "hole" (as resulting from the corresponding composition of cobordisms, $8=\square 0 \square$.

\section{Homotopy pushouts and finite 1-dimensional CW-complexes}

The general idea of homotopy pushouts and homotopy quotients, which has become an essential slogan in higher category theory [1, and more recently in type theory [9], is that instead of equating elements, one sews in a path between them, so as to keep track of the fact that two elements could be equal in more than one way. When applied to the composition of cospans, the homotopy-pushout operation matches well with the various interpretations of cospans in applications: to connect two "devices", draw "cables" from the outputs of the first to the inputs of the second.

Let $X$ and $Y$ be topological spaces, and let $A$ be a finite set, considered as a discrete space. The homotopy pushout $X \amalg_{A} Y$ of a span $X \stackrel{f}{\leftarrow} A \stackrel{g}{\rightarrow} Y$,

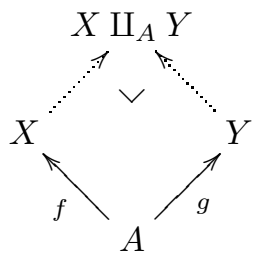

is obtained by attaching an interval in $X \amalg Y$ from $f(a) \in X$ to $g(a) \in Y$, for each point $a \in A$. For example, the homotopical version of the pushout in (2) is shown below, clearly corresponding to 0 :

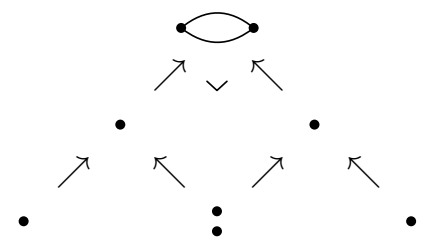

We see in particular, that even when the spaces $X, Y$, and $A$ are all discrete, the homotopy pushout $X \amalg_{A} Y$ will not in general be discrete. To accommodate the homotopy pushout, we need a category 
of cospans with discrete endpoints, but with apex at least a homotopy 1-type, say a 1-dimensional CW complex. Recall that a finite 1-dimensional $C W$-complex is a space obtained from a finite set of points by attaching a finite set of segments by their endpoints. The homotopy pushout described above is a paradigmatic example. A finite 1-dimensional CW-complex is thus a topological graph; if it is connected it is characterized up to homotopy by its first Betti number, the number of independent cycles. Any connected finite 1-dimensional CW-complex is therefore homotopy equivalent to a bouquet of circles. Note that the homotopy pushout is a homotopy-invariant construction: replacing any of the three objects in the span $X \leftarrow A \rightarrow Y$ by something homotopy equivalent will not change the homotopy class of the homotopy pushout.

Let Cospan denote the symmetric monoidal category whose objects are finite sets, and whose arrows are homotopy classes of cospans $A_{0} \rightarrow X \leftarrow A_{1}$, where $X$ is a finite 1-dimensional CW-complex. The monoidal structure is given by disjoint union. It is easy to see that such cospans are closed under composition given by homotopy pushout, since this operation attaches 1-cells, but nothing higher-dimensional. Since we only consider cospans up to homotopy, it is also clear that composition is associative and unital.

\section{Homotopy cospans and cobordisms}

Theorem. The symmetric monoidal category Cospan, whose objects are finite sets and whose morphisms are homotopy classes of cospans, is the free symmetric monoidal category on a commutative Frobenius object.

The universal property can be established directly, without reference to cobordisms, by copying over the proof in [6] almost verbatim. This is possible since the generators and relations of Cospan as a symmetric monoidal category are readily seen to correspond exactly to the axioms for commutative Frobenius algebras. The key point here is that the invariants detecting whether two connected cospans are homotopic are the same as those classifying connected 2-cobordisms, as used in [6].

It is also possible to establish this result without mention of generators and relations, by means of a geometric formalization of the analogy between cospans and cobordisms, establishing an equivalence of symmetric monoidal categories Cospan $\simeq \mathbf{2 C o b}$. This provides an alternative proof of the Theorem, since $\mathbf{2 C o b}$ is already known to have the universal property ([6], Theorem 3.6.19).

We first need to enhance the geometric realization of a cospan $A_{0} \rightarrow X \leftarrow A_{1}$, by separating out its input and output points. We do that systematically by replacing $X$ by the mapping cylinder of $A_{0}+A_{1} \rightarrow X$; this amounts to attaching a 1-cell from each point in $A_{0}+A_{1}$ to its image point in $X$, and produces thus a homotopy equivalent graph whose input and output points are all pairwise disjoint.

As an example, here is the replacement for the cospan representing multiplication:

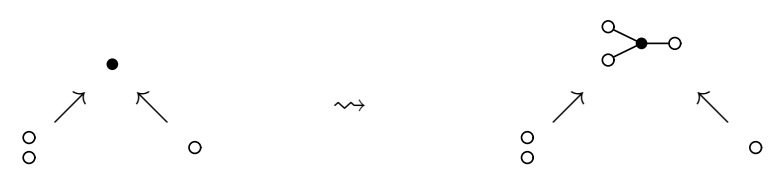

already considerably strengthening the analogy with 5 . (At this point it is worth recalling that a cobordism is also in fact a kind of cospan (cf. [6], §1.2): it is the surface with boundary, together with specific inclusions $\Sigma_{0} \rightarrow M \leftarrow \Sigma_{1}$ from closed 1-manifolds onto the boundary.)

With this preparatory mapping-cylinder step, we can describe the correspondence geometrically in Euclidean 3-space. Given a cospan we choose a suitable embedding of the mapping-cylinder graph into $\mathbf{R}^{2} \times[0,1] \subset \mathbf{R}^{3}$, with its input points at level 0 and output points at level 1 . By suitable, we mean: smooth on each 1-cell, and at each vertex the adjacent 1-cells should not share tangent directions. Other than these requirements, it is not important how exactly the embedding is arranged - even the isotopy class does not matter. In order to construct a cobordism from this, we first take the boundary of a tubular neighborhood of the embedded graph as a subset of $\mathbf{R}^{3}$; specifically we can take the set of points in $\mathbf{R}^{3}$ 
at fixed distance $\epsilon>0$ from the graph. If $\epsilon$ is chosen small enough, which is possible by compactness of the finitely many closed cells, the resulting subset will be a topological surface. We intersect this surface with $\mathbf{R}^{2} \times[0,1]$ to obtain a cobordism, whose boundary consists of small circles around the input and output points of the graph.

Conversely, any cobordism can be embedded in $\mathbf{R}^{2} \times[0,1]$ with in-boundaries at level 0 and outboundaries at level 1. Its interior admits a deformation retract onto a graph, commuting (at the boundary levels $\mathbf{R}^{2} \times\{0,1\}$ ) with the deformation retract of the open disks onto their center points; the result is unique up to homotopy. With boundary points determined as those at levels 0 and 1 , this defines a cospan. It is clear that these two constructions are mutually inverse (up to the equivalences in question: homotopy equivalence of graph cospans, and homeomorphism rel the boundary for cobordisms).

Finally, it is clear from the geometry that composition of cospans corresponds precisely to composition of cobordisms: Given composable cospans $A_{0} \rightarrow X \leftarrow A_{1}$ and $A_{1} \rightarrow Y \leftarrow A_{2}$, the composite given by the homotopy pushout can be realized geometrically by embedding (the mapping cylinders of) $X$ into $\mathbf{R}^{2} \times[0,1]$ and $Y$ into $\mathbf{R}^{2} \times[2,3]$ and sewing in 1-cells $A_{1} \times I$ in $\mathbf{R}^{2} \times[1,2]$. Cobordisms can be composed in exactly the same way, by sewing in cylinders in $\mathbf{R}^{2} \times[1,2]$ between the boundary circles at level 1 and 2 .

Acknowledgments. Kock was supported by grant MTM2013-42293-P of Spain, and Spivak was supported by AFOSR grant FA9550-14-1-0031 and NASA grant NNH13ZEA001N. We thank the referee for useful remarks.

\section{References}

[1] J. C. Baez and J. Dolan. From finite sets to Feynman diagrams. In Mathematics unlimited-2001 and beyond, pp. 29-50. Springer-Verlag, Berlin, 2001. arXiv:math.QA/0004133

[2] J. C. BAEZ and B. Fong. A Compositional Framework for Passive Linear Networks. arXiv:1504.05625.

[3] B. CoyA and B. Fong. Corelations are the prop for extraspecial commutative Frobenius monoids. arXiv:1601.02307.

[4] J. Fuchs, I. Runkel and C. Schweigert. Conformal correlation functions, Frobenius algebras and triangulations. Nuclear Phys. B 624 (2002), 452-468.

[5] F. Gadducci and R. Heckel. An inductive view of graph transformation. In Recent trends in algebraic development techniques (Tarquinia, 1997), vol. 1376 of Lecture Notes in Comput. Sci., pp. 223-237. Springer, Berlin, 1998.

[6] J. Kock. Frobenius algebras and 2D topological quantum field theories. No. 59 in London Mathematical Society Student Texts. Cambridge University Press, Cambridge, 2003.

[7] S. LACK. Composing PROPS. Theory Appl. Categ. 13 (2004), No. 9, 147-163.

[8] R. Rosebrugh, N. Sabadini, and R. F. C. Walters. Generic commutative separable algebras and cospans of graphs. Theory Appl. Categ. 15 (2005/06), No. 6, 164-177.

[9] The Univalent Foundations Program. Homotopy type theory-univalent foundations of mathematics. The Univalent Foundations Program, Princeton, NJ; Institute for Advanced Study (IAS), Princeton, NJ, 2013. Available from http://homotopytypetheory.org/book.

\footnotetext{
J. Kock, Departament de Matemàtiques, Universitat Autònoma de Barcelona, SPAin

E-mail address: kock@mat.uab.cat

D.I. Spivak, Department of Mathematics, Massachusetts Institute of Technology, USA

E-mail address: dspivak@gmail.com
} 